\title{
Proteomic analysis of the cerebrospinal fluid of Parkinson's disease patients
}

Cell Research (2009) 19:1401-1403. doi: 10.1038/cr.2009.131; published online 1 December 2009

\section{Dear Editor,}

Parkinson's disease (PD) is the second most common neurodegenerative disease after Alzheimer's disease (AD). Although treatments for PD may be beneficial in the early stages of disease, accurate diagnosis during these stages remains a challenge. An ideal diagnosis for PD should be highly specific and sensitive, as well as be able to predict disease progression. Currently, diagnosis of PD relies mainly on the evaluation of clinical features, specifically cardinal signs. However, accurate diagnosis on the basis of clinical features is partially hampered by the existence of other forms of Parkinsonism, including corticobasal degeneration (CBD), dementia with Lewy bodies (DLB), multisystem atrophy (MSA) and progressive supranuclear palsy (PSP). Non-motor features, such as dementia and dysautonomia, occur frequently, especially in the advanced stages of PD. These factors complicate the differentiation of PD from CBD, DLB, MSA and PSP [1]. Thus, there is currently great interest in the development of more effective procedures for the diagnosis of PD in its early stages [2]. In the present study, we performed two-dimensional gel electrophoresis (2-DE)-based proteomic analysis of CSF from PD patients. Up to 14 differentially expressed proteins were identified by liquid chromatography in combination with tandem mass spectrometry (LC-MS/MS). Our study provides a group of interesting candidate molecules for consideration as potential diagnostic markers of PD.

Collection of PD and AD CSF samples and the experimental procedures for 2-DE and LC-MS/MS analysis are described in the Supplementary information, Data S1.

To investigate alterations in protein expression associated with $\mathrm{PD}$, we performed comparative high-resolution 2-DE experiments on protein samples pooled from PD or control patients using immobilized $\mathrm{pH}$ gradient strips ( $\mathrm{pH} \mathrm{4-7,} 24 \mathrm{~cm}$ length). The overall protein expression patterns in the gels were very similar between PD and control groups. Figure S1 in Supplementary information shows a typical image of a silver-stained gel containing separated CSF proteins. The highest number of detected spots in a single gel containing a specific PD CSF sample was 930 . The intensity of each protein spot in gels from each group was quantified; these intensities were then compared between PD and controls. Only the ratios of normalized spot intensities between PD and control groups were calculated, and spots showing more than two fold difference with statistical significance $(P<0.05)$ were selected for LC-MS/MS analysis. The relative levels of 21 protein spots (from a mean of five gels for each group), including eight spots that were upregulated and 13 that were down-regulated in PD CSF, were determined. These spots corresponded to 14 different polypeptides (Supplementary information, Table S1), among which five proteins were represented by at least two distinct spots on the 2-DE gels. 2-DE gel maps of 11 up- or down- regulated proteins are shown in Figure 1.

As shown in the Supplementary information, Figure S2, the levels of ApoE, autotaxin and PEDF in pooled PD samples were 2.5 10-fold higher than in control samples, whereas the level of $\mathrm{C} 4 \alpha$ was reduced by $79 \%$ in PD CSF compared with control CSF. These results were consistent with the data from 2D gel-based proteomic profiling in the present study (Supplementary information, Table S1). To determine whether the changes observed in pooled samples also occurred in individual PD patients, CSF samples from a new set of subjects consisting of 14 PD patients and 12 controls were analyzed by western blotting. The average levels of ApoE and autotaxin in 14 individual PD patients were increased by $3.1-$ and 1.8 fold, respectively, relative to the levels in controls. By contrast, the average level of $\mathrm{C} 4 \alpha$ in PD patients was $\sim 34 \%$ of the control level (Supplementary information, Figure S3). Although there was a trend of increased levels of PEDF in PD CSF relative to control levels, this difference was not statistically significant (Supplementary information, Figure S3B).

To further investigate whether the levels of ApoE, autotaxin, PEDF and C4 $\alpha$ observed in PD CSF can be used to distinguish $\mathrm{PD}$ from $\mathrm{AD}$, the levels of these proteins were also examined by western blotting of a set of CSF samples from seven $\mathrm{AD}$ patients and eight controls. The 


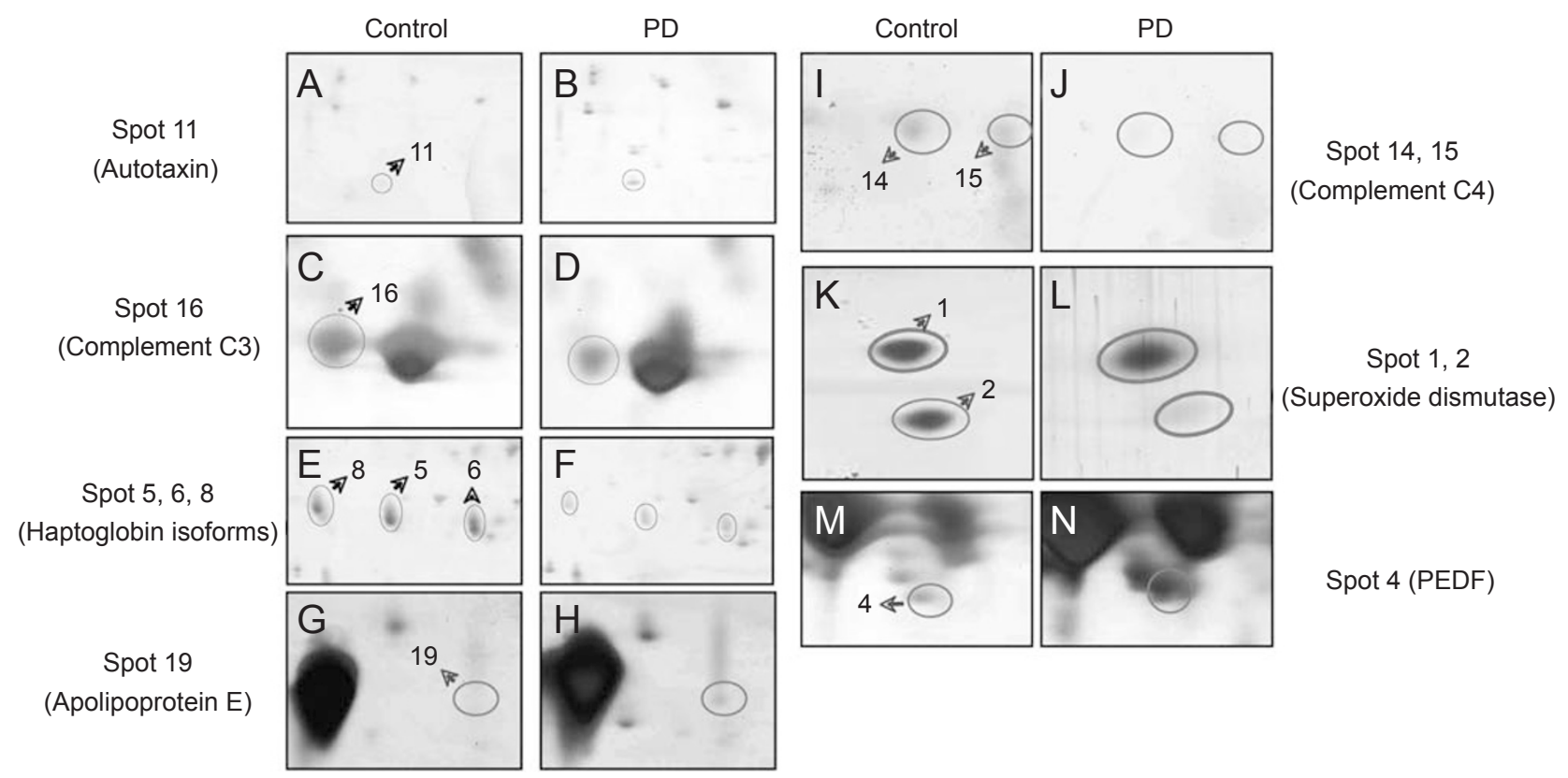

Figure 1 Representative 2-DE gel protein spots displaying differential expression levels in CSF from PD patients and controls. The proteins autotaxin (A-B, spot 11 in Figure 1), ApoE (G-H, spot 19) and PEDF (M-N, spot 4) were increased in abundance in the CSF from PD patients, relative to the levels in controls, whereas the levels of complement C3 (C-D, spot 16), three isoforms of haptoglobin (E-F, spots 5, 6 and 8) and C4a (I-J spots 14 and 15) were decreased in PD CSF relative to the levels in controls. Representative 2-DE gel protein spots for SOD1 are also shown in K-L. Please note that owing to distortion of the lower parts of the original 2-DE gels, spot 2 was slightly shifted.

average levels of ApoE and $\mathrm{C} 4 \alpha$ in the seven individual $\mathrm{AD}$ patients were up to 4.0- and 1.8-fold higher than those in controls, respectively, whereas the average levels of PEDF and autotaxin in AD CSF were $32.4 \%$ and $25.6 \%$ of those in controls, respectively (Supplementary information, Figure S4). Thus, autotaxin, PEDF and C4 $\alpha$ showed contradictory changes in AD and PD CSF.

As demonstrated in Figure 1, the levels of two isoforms of SOD1 were found to change in different fashions in PD CSF. To better characterize the differential expression of these two isoforms of SOD1 in the context of pathological conditions, we carried out 2-DE analysis of $80 \mu \mathrm{g}$ of proteins from each of PD and AD CSF samples (Supplementary information, Figure S5). Western blotting of 2-DE gels showed that four protein spots (designated spots 1-4) were specifically recognized by SOD1 antibodies (Supplementary information, Figure $\mathrm{S} 5 \mathrm{~A})$. Comparison of the immunoblots with the corresponding area in silver-stained sister gels showed that the four SOD1-immunoreactive spots matched spots on silver-stained gels that had previously been identified by LC-MS/MS as SOD1 isoforms (Supplementary information, Figure S5A vs Figure S5B). Based on the estimated $\mathrm{p} I$ and $\mathrm{MW}$ values of the four SOD1 isoforms, the two spots identified as SOD1 in Figure 1 corresponded to spots 1 and 2 shown in the Supplementary information, Figure S5.

Moreover, we found that the $17.0 \mathrm{kDa}$ isoform $(\mathrm{p} I 5.9$, spot 1), but not the other three isoforms, showed strong immunoreactivity to anti-DNP antibody in PD CSF samples, indicating that this SOD1 isoform is oxidatively modified by carbonyl formation in PD patients (Supplementary information, Figure S6C-S6D). By contrast, oxidatively modified SOD1 was not detected in control CSF samples (Supplementary information, Figure S6AS6B). Interestingly, no DNP-immunoreactive spots were detectable by western blotting in a pooled CSF sample from seven $\mathrm{AD}$ patients, indicating that the level of oxidative modification of SOD1 isoforms in AD CSF is very low (Supplementary information, Figure S6E-S6F). Furthermore, SOD1 isoforms (spots 1-4) in PD CSF samples did not react with antibodies that detect serine/threonine phosphorylation and tyrosine nitration (data not shown).

In the present study, we used high-resolution 2-DE and MS to identify 21 differentially expressed proteins (including isoforms of the same proteins in some cases) in the CSF of PD patients. Alterations in the levels of ApoE, autotaxin, complement $\mathrm{C} 4 \alpha$ and SOD1 may be 
the results of pathological changes, thus providing new insight into the pathogenic process of PD. Our study demonstrates for the first time that the level of autotaxin is up-regulated in PD CSF (spot 11 in Figure 1A). Autotaxin belongs to the nucleotide pyrophosphatase family of ectoenzymes and exoenzymes. It was recently found to be identical to lysophospholipase $\mathrm{D}$, which is the major enzyme involved in the generation of lysophosphatidic acid (LPA) [3-5]. LPA promotes growth, differentiation, survival and motility in many different cell types [3]. Elevated autotaxin levels probably lead to the production of more LPA, which in turn promotes cell survival and proliferation.

Of further interest, we also report here that the levels of SOD1 are altered in CSF of PD patients. Our data demonstrate that these alterations in the levels of SOD1 isoforms are unique to PD CSF [6].

In conclusion, our findings suggest that most of the differentially regulated proteins in PD CSF appear to be associated with the pathologies and metabolic pathways that are also involved in other neurodegenerative diseases. Our finding that ApoE is elevated in both $\mathrm{PD}$ and $\mathrm{AD}$ CSF supports the view that these two diseases may share common pathogenic mechanism(s) that could potentially be targeted by similar therapeutic strategies. Given that the results presented here were obtained from a relatively small number of samples, elucidation of their clinical significance requires further investigations.

\section{Acknowledgments}

This work was supported by grants from the Chinese Ministry of Science \& Technology (No. 2004AA221130), the Shanghai Metropolitan Fund for Research and Development (No. 07DJ14005), the National Natural Science Foundation of China (Nos. 30525041, 30721004), and the State Key Program for Basic Research of China (No. 2006CB500704).

Jiguang Guo ${ }^{1,2}$, Zhongwu Sun ${ }^{3}$, Shifu Xiao ${ }^{4}$, Dongping Liu ${ }^{1}$, Guohua Jin ${ }^{5}$, Ersong Wang ${ }^{6}$, Jiangning Zhou $^{7}$, Jiawei Zhou ${ }^{2}$
${ }^{1}$ Laboratory of Molecular Cell Biology, Institute of Biochemistry and Cell Biology; ${ }^{2}$ Institute of Neuroscience, State Key Laboratory of Neuroscience, Shanghai Institutes for Biological Sciences, Chinese Academy of Sciences, Shanghai 200031, China; ${ }^{3}$ Department of Neurology, the First Affiliated Hospital, Anhui Medical University, Hefei 230022, China; ${ }^{4}$ Department of Geriatric Psychiatry, Shanghai Mental Health Center, Shanghai 200030, China; ${ }^{5}$ Department of Anatomy, Nantong University School of Medicine, Nantong, JS 226001, China; ${ }^{6}$ Department of Neurosurgery, Huashan Hospital, Shanghai 200040, China; ${ }^{7}$ Department of Neurobiology, University of Science and Technology of China, Hefei, Anhui, 230022, China Correspondence: Jiawei Zhou

Tel/Fax: +86-21-5492 1073

E-mail: jwzhou@ion.ac.cn

\section{References}

1 Wenning GK, Ebersbach G, Verny M. Progression of falls in postmortem-confirmed parkinsonian disorders. Mov Disord 1999; 14:947-950.

2 Abdi F, Quinn JF, Jankovic J, et al. Detection of biomarkers with a multiplex quantitative proteomic platform in cerebrospinal fluid of patients with neurodegenerative disorders. $J$ Alzheimers Dis 2006; 9:293-348.

3 Tokumura A, Majima E, Kariya Y, et al. Identification of human plasma lysophospholipase D, a lysophosphatidic acidproducing enzyme, as autotaxin, a multifunctional phosphodiesterase. J Biol Chem 2002; 277:39436-39442.

4 Moolenaar WH. Lysophospholipids in the limelight: autotaxin takes center stage. J Cell Biol 2002; 158:197-199.

5 Van Meeteren LA, Ruurs P, Stortelers C, et al. Autotaxin, a secreted lysophospholipase D, is essential for blood vessel formation during development. Mol Cell Biol 2006; 26:50155022

6 Choi J, Rees HD, Weintraub ST, Levey AI, Chin LS, Li L. Oxidative modifications and aggregation of $\mathrm{Cu}, \mathrm{Zn}$-superoxide dismutase associated with Alzheimer and Parkinson diseases. $J$ Biol Chem 2005; 280:11648-11655.

(Supplementary information is linked to the online version of the paper on the Cell Research website.) 